\title{
Surface hardness affects anatomical parameters of dorsal digital artery of cattle
}

Ronaldo Gomes Gargano;, Guilherme Bonafin de Souza, Bruna Stanigher Barbosa, Fábio Parra Sellera, Carolina dos Anjos, Edlen de Andrade Medeiros, Fernando José Benesi, Stefano Carlo Filipo Hagen, Fabio Celidonio Pogliani

Faculdade de Medicina Veterinária e Zootecnia, Universidade de São Paulo (USP), São Paulo, SP, Brazil

${ }^{*}$ Corresponding author

e-mail: rggargano@usp.br

\section{Abstract}

Lameness is the major concern in dairy industry that negatively affects the well-being of cattle, generating losses in milk production. Some studies have been shown that when dairy cow's comfort is compromised, it increases the standing time in free stall alleys resulting in claw horn disruption lesions due to the constant compression of the corium tissue. On hard surfaces, there is no dissipation of the force applied to the ground which results in a ground reaction force with the same intensity and opposite direction, increasing the pressure inside the claw capsule. Therefore, our hypothesis is that the anatomy and vascular indexes of dorsal digital artery of the cattle, the main arterial blood supply of the bovine digits, are influenced by the consistency of the surface bearing the hind limbs. In the study seven healthy Holstein steers, 16-20 months old, $254.4 \pm 15.7 \mathrm{~kg}$, were used. All the animals were kept in standing position and placed in a hard concrete and in a soft wood shaving floor, with $10 \mathrm{~cm}$ of thickness. Ultrasonographic exams began after 30 minutes and were performed on the dorsal view of randomly chosen pelvic limbs, at the proximal interphalangeal joint. Anatomical B-mode measurements and vascular indexes obtained by pulsed Doppler evaluations were performed. Doppler outputs were done by three spectral waves and the exam was repeated three times in each limb. Statistical analysis considered the residuals normal plot and used paired-samples T test, verifying the acceptance of the alternative hypothesis at the significance level of 5\%. All the animals were well-adapted to the management, remained calm without the use of tranquilizers and did not need physical restraints in the pelvic limbs. The soft wood shaving floor increased the diameter and cross sectional area of the dorsal digital artery, being statistically significant when compared to the hard surface floor. Hypothetically, this effect is due to the greater dissipation of the weight force on the soft surface which decreases the ground reaction force. The resistivity and pulsatility indexes showed no significant differences. The blood flow volume was higher in the soft wood shaving surface than in the harder one, however that difference was 
not statistically significant. Based on these results, the consistency of the bearing surface can influence the diameter and cross sectional area of the dorsal digital artery of the cattle. 